\title{
Porous hydrogel encapsulated photonic barcodes for multiplex detection of cardiovascular biomarkers
}

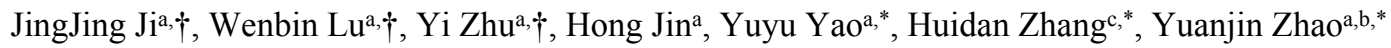

${ }^{a}$ Department of Cardiology, ZhongDa Hospital Affiliated with Southeast University, Nanjing, China

Email: yaoyuyunj@hotmail.com

${ }^{b}$ State Key Laboratory of Bioelectronics, school of Biological science and Medical Engineering. Southeast

University,Nanjing, China

Email: yjzhao@seu.edu.cn

'School of Engineering and Applied Sciences and Department of Physics, Harvard University, Cambridge, Massachusetts 02138, United States

Email: hdzhang@seas.harvard.edu

$\dagger$ These authors contributed equally to this work 

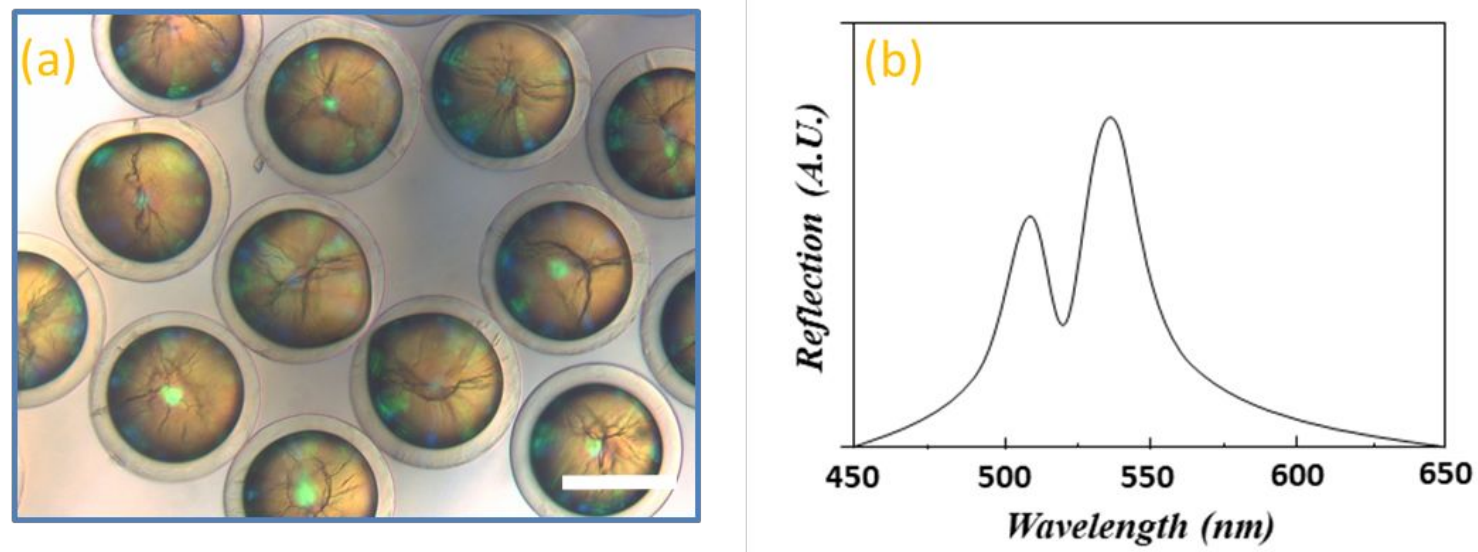

Figure S1. Optical characterization of the core-shell PhC particles. (a) Optical image and (b) reflection spectrum of core-shell PhC particles with two reflection peaks. Scale bar is $200 \mu \mathrm{m}$.
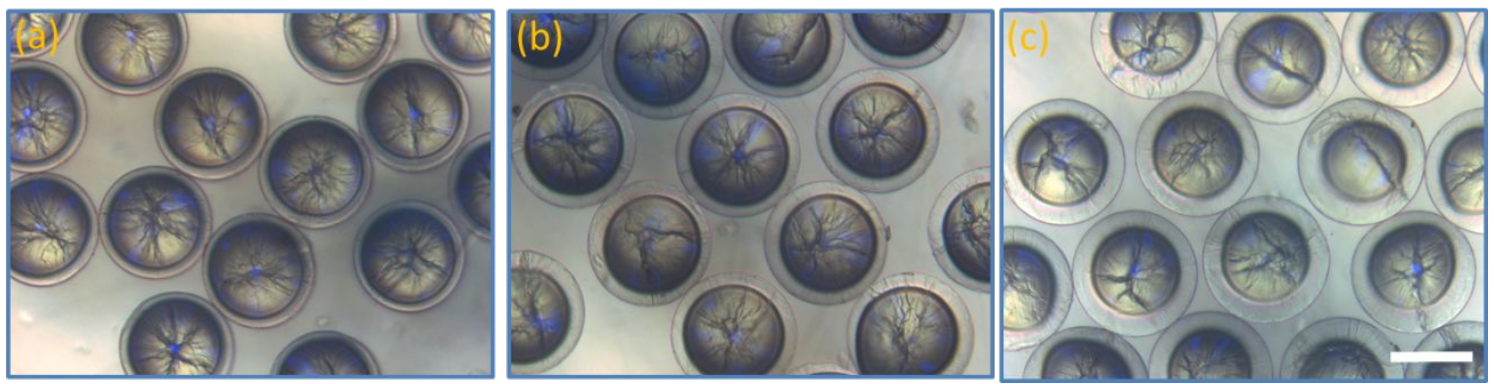

Figure S2. Optical images of the core-shell PhC particles with a transparent shell layer structure. (a) ething time with $10 \mathrm{~min}$, (b) ething time with $20 \mathrm{~min}$, (c) ething time with $30 \mathrm{~min}$,Scale bar is $200 \mu \mathrm{m}$. 